\title{
Coupling between clathrin-dependent endocytic budding and F-BAR-dependent tubulation in a cell-free system
}

Min Wu, Bo Huang, Morven Graham, Andrea Raimondi, John E. Heuser, Xiaowei Zhuang and Pietro De Camilli

Nat. Cell Biol. 12, 902-908 (2010); published online 22 August 2010; corrected after print, 26 August 2010

In the version of this letter initially published online and in print, the author affiliations were incorrect. The correct affiliations are:

Min $\mathrm{Wu}^{1,2,4}$, Bo Huang ${ }^{5,8,9}$, Morven Graham² ${ }^{2}$ Andrea Raimondi ${ }^{1,2,4}$, John E. Heuser ${ }^{7}$, Xiaowei Zhuang ${ }^{5,6,8}$ and Pietro De Camilli ${ }^{1,2,3,4,10}$

${ }^{1}$ Howard Hughes Medical Institute, Yale University School of Medicine, New Haven, CT 06520, USA. ${ }^{2}$ Department of Cell Biology, Yale University School of Medicine, New Haven, CT 06520, USA. ${ }^{3}$ Department of Neurobiology, Yale University School of Medicine, New Haven, CT 06520, USA. ${ }^{4}$ Program in Cellular Neuroscience, Neurodegeneration and Repair, Yale University School of Medicine, New Haven, CT 06520, USA. ${ }^{5}$ Department of Chemistry and Chemical Biology, Harvard University, Cambridge MA 02138, USA. ${ }^{6}$ Department of Physics, Harvard University, Cambridge MA 02138, USA. 'Department of Cell Biology and Physiology, Washington University School of Medicine, 660 South Euclid Avenue, St. Louis, MO 63110, USA. ${ }^{8}$ Howard Hughes Medical Institute, Harvard University, Cambridge MA 02138 , USA. ${ }^{9}$ Current address: Department of Pharmaceutical Chemistry, Department of Biochemistry and Biophysics, University of California, San Francisco, CA 94158 ,

USA. ${ }^{10}$ Correspondence should be addressed to P.D.C. (e-mail: pietro.decamilli@yale.edu)

This error has been corrected in both the HTML and PDF versions of the letter.

\section{Meiotic kinetochores get pushed aside by a CLS act}

\section{Xue Han and Martin Srayko}

Nat. Cell Biol. 12, 849-851 (2010); published online 22 August 2010; corrected after print, 2 September 2010

In the version of this News and views article initially published online and in print, "congression" has replaced "orientation" in the following sentence:

Interestingly, the authors also showed that chromokinesin KLP-19, a non-kinetochore motor-protein required for the proper congression and alignment of mitotic chromosomes ${ }^{11}$, was dispensable for proper meiotic chromosome congression before anaphase.

This error has been corrected in both the HTML and PDF versions of the letter.

\section{Plasma membrane contributes to the formation of pre-autophagosomal structures}

\section{Brinda Ravikumar, Kevin Moreau, Luca Jahreiss, Claudia Puri and David C. Rubinsztein}

Nat. Cell Biol. 12, 747-757 (2010); published online 18 July 2010; corrected after print, 16 September 2010

In the version of this article initially published online and in print, the cholera toxin in Fig 8d was inadvertently placed outside of the internalised vesicles rather than inside the vesicles. Since the authors used cholera toxin as a tracer for internalised plasma membrane, this error does not affect their model for autophagosome precursor formation. The correct version of this figure is shown to the right. This error has been corrected in both the HTML and PDF versions of the article.

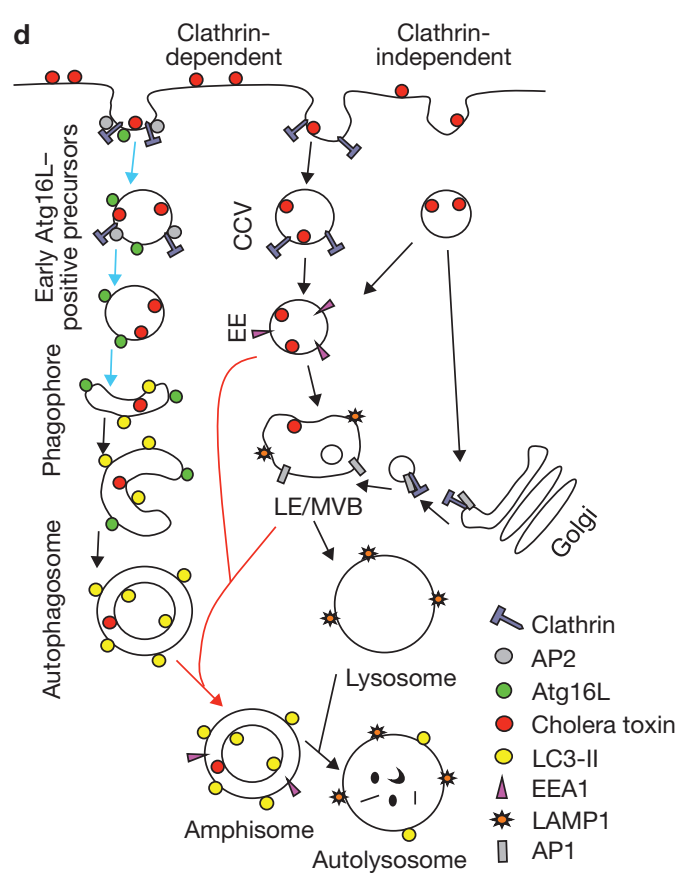

